SUPPORTING INFORMATION

\title{
Aligned Nanofibers from Polypyrrole/Graphene as Electrodes for Regeneration of Optic Nerve via Electrical Stimulation
}

Lu Yan, ${ }^{a}$ Bingxin Zhao, ${ }^{a}$ Xiaohong Liu, ${ }^{a}$ Xuan Li, ${ }^{a}$ Chao Zeng, ${ }^{b}$ Haiyan Shi, ${ }^{a}$ Xiaoxue $\mathrm{Xu},{ }^{\mathrm{c}}$ Tong Lin, ${ }^{\mathrm{b}}$ Liming Dai, ${ }^{\mathrm{a}, \mathrm{d},{ }^{*}}$ and Yong $\operatorname{Liu}^{\mathrm{a}, \mathrm{e}, *}$

${ }^{a}$ Lab of Nanoscale Biosensing and Bioimaging, Institute of Advanced Materials for Nano-Bio Applications, School of Ophthalmology \& Optometry, Wenzhou Medical University, Wenzhou, Zhejiang 325027, China

${ }^{b}$ Institute for Frontier Materials, Deakin University, Waun Ponds, VIC 3217, Australia.

${ }^{c}$ Centre for Biomedical Materials and Engineering, Harbin Engineering University, Harbin, Heilong Jiang 150001, China

${ }^{d}$ Center of Advanced Science and Engineering for Carbon (Case4Carbon), Department of Macromolecular Science and Engineering, Case Western Reserve University, Cleveland, Ohio 44106, United States.

${ }^{e}$ Department of Chemistry and Biomolecular Science, Macquarie University, Sydney, NSW 2109, Australia.

*Emails: liming.dai@case.edu, yongliu1980@hotmail.com 
Energy Dispersive Spectroscopy (EDS)

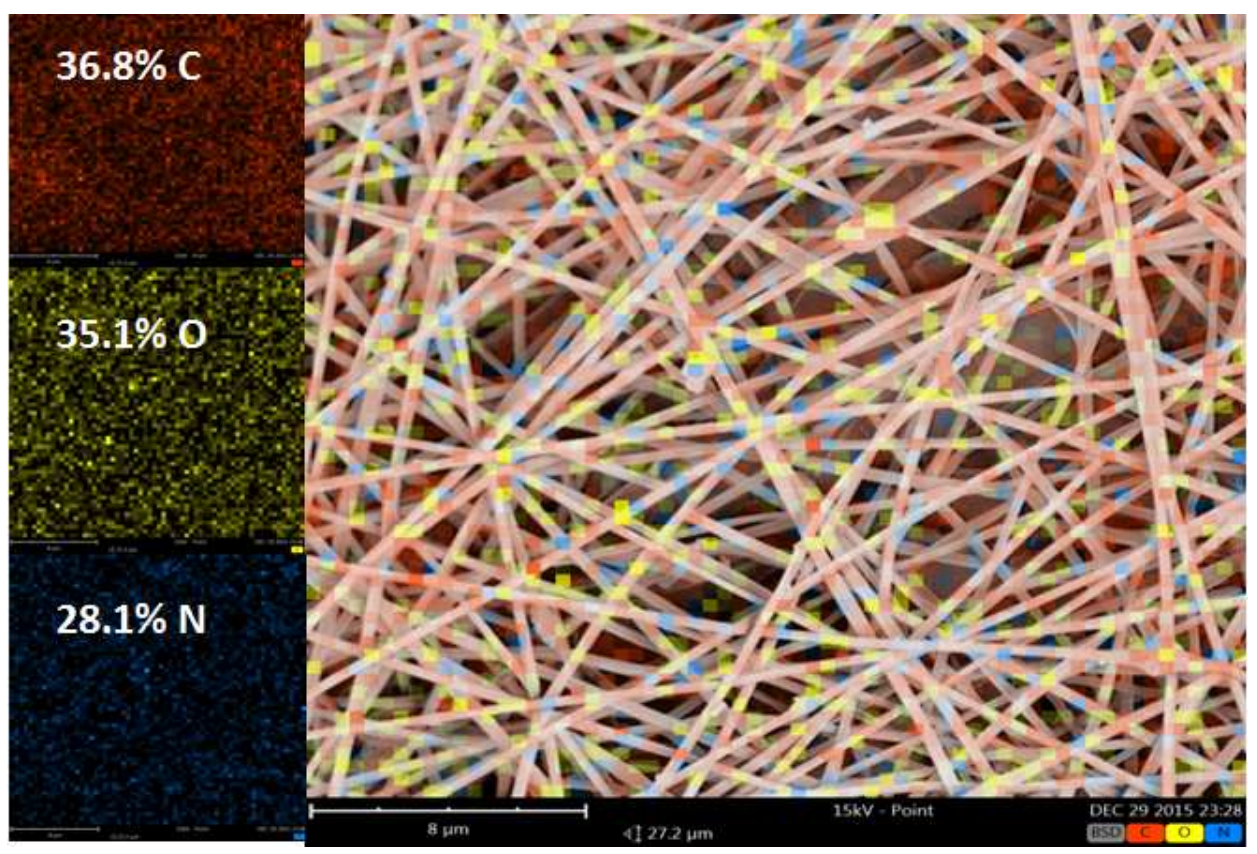

Figure S1. SEM image and elemental analysis on the aligned nanofibers from $6 \%$ (w/w) PPy-G/PLGA determined by the Energy Dispersive Spectroscopy (Phenom Prox). 
Thermal gravimetric analysis (TGA)

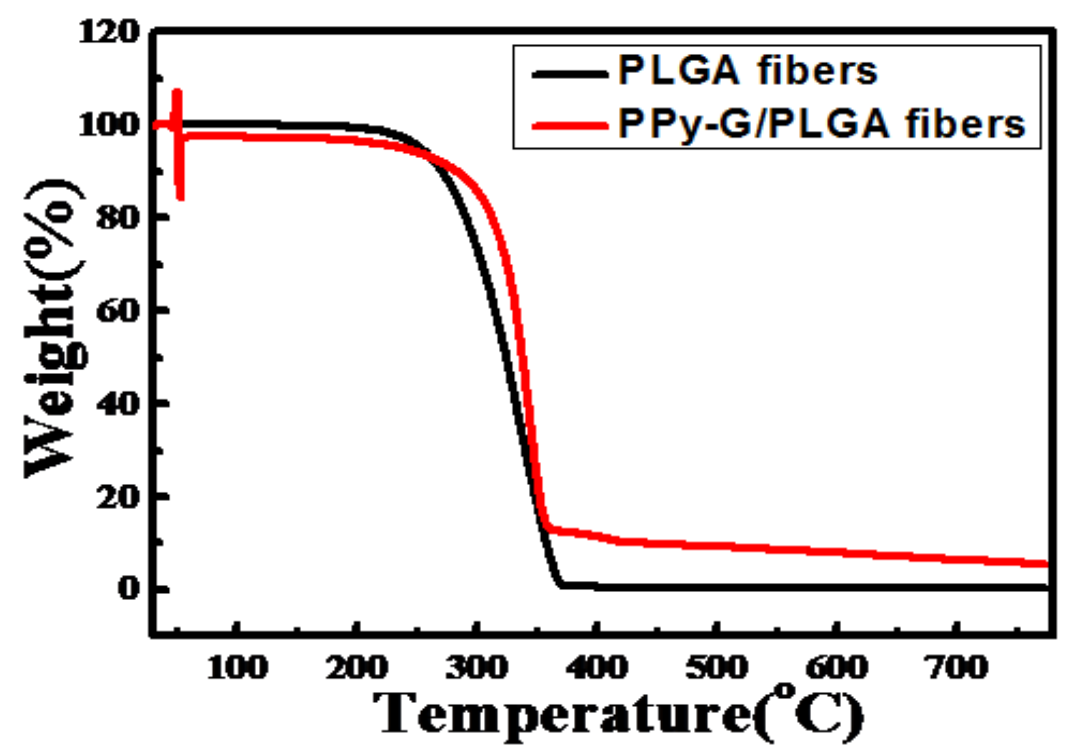

Figure S2. TGA profile of the PPy-G/PLGA nanofibers compared to that of the PLGA nanofibers.

Cyclic voltammetry

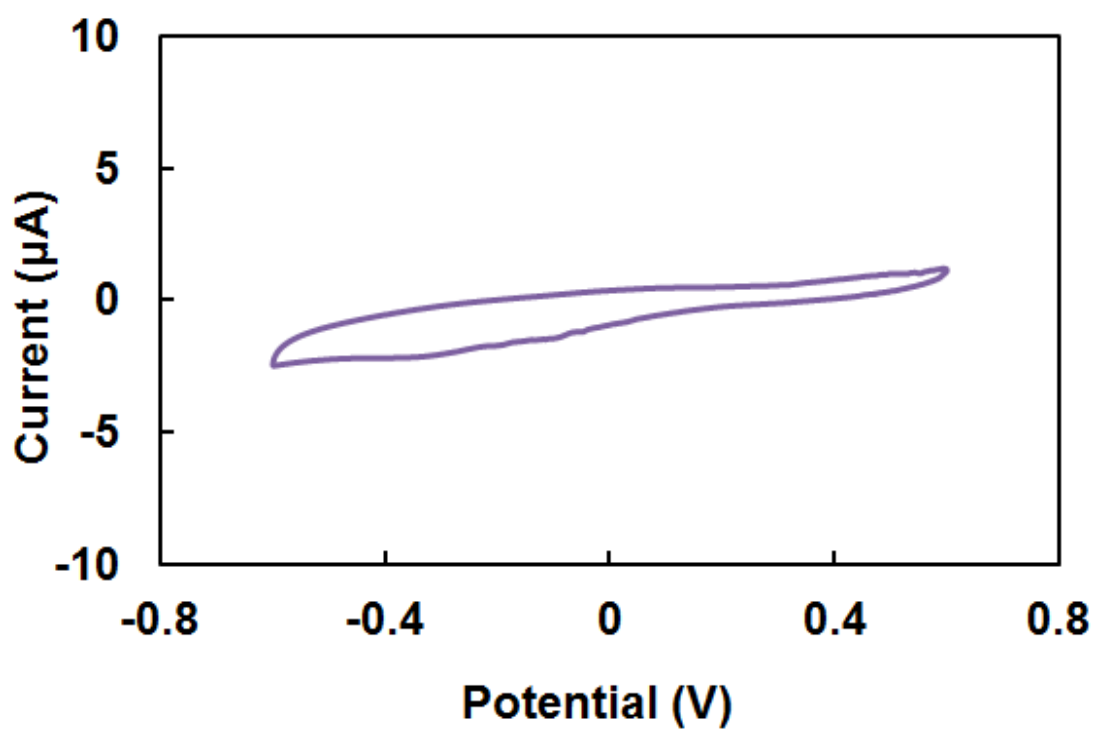

Figure S3. Cyclic voltammogram in $0.1 \mathrm{M}$ PBS solution $(\mathrm{pH}=7.4)$ at the graphite after ball milling without addition of pyrrole. 


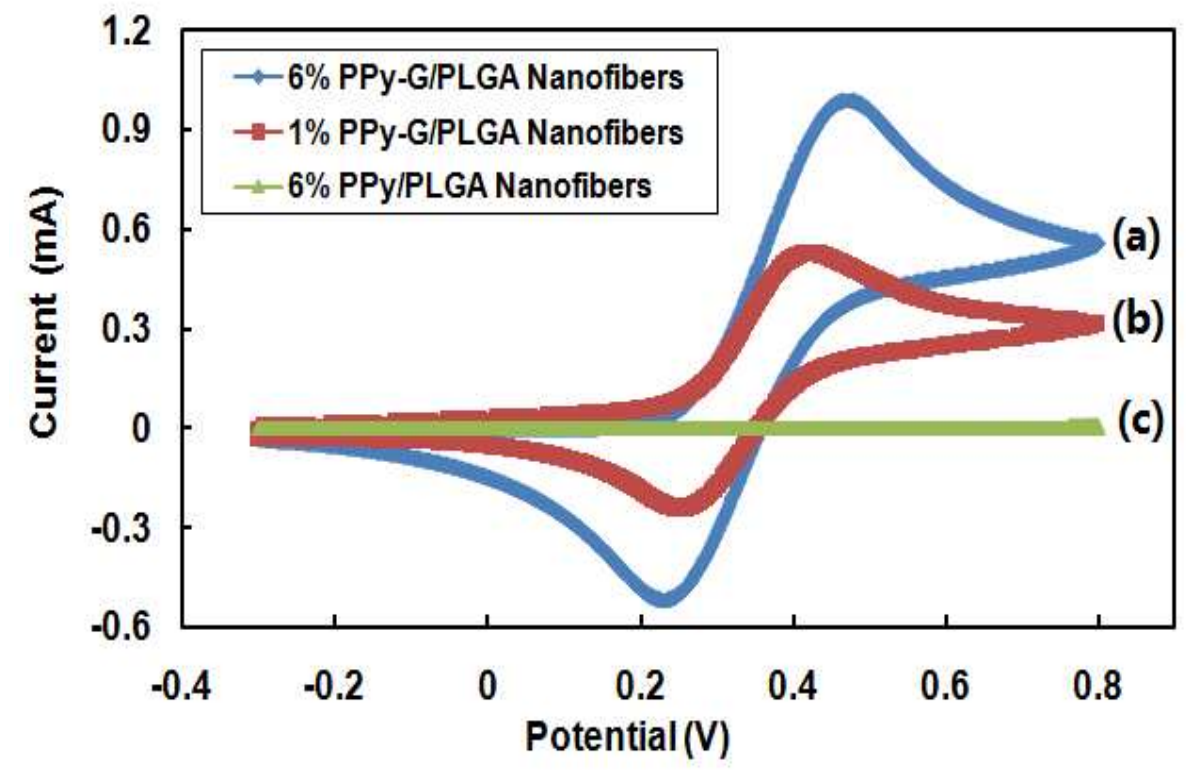

Figure S4. (a) Cyclic voltammograms recorded in $10 \mathrm{mM} \mathrm{K}_{4} \mathrm{Fe}(\mathrm{CN})_{6} / 0.1 \mathrm{M}$ PBS solution at (a) the 6\% PPy-G/PLGA aligned nanofibers, (b) the 1\% PPy-G/PLGA aligned nanofibers, and (c) the 6\% PPy/PLGA aligned nanofibers for comparison. Electrode size: $1 \mathrm{~cm}^{2}$. Scan rate: $50 \mathrm{mV} / \mathrm{s}$.

\section{Selection of the potential for electrical stimulation}

In order to identify the best applied voltage for ES of RGCs, different voltages have been investigated. RGCs were subsequently stimulated on the pristine ITO glass at various step constant potentials including $\pm 1000 \mathrm{mV} / \mathrm{cm}, \pm 700 \mathrm{mV} / \mathrm{cm}, \pm 400 \mathrm{mV} / \mathrm{cm}$, and $\pm 100 \mathrm{mV} / \mathrm{cm}$, respectively. As shown in Figure S5, cell morphology didn't change too much when the step potential was pulsed between $\pm 400 \mathrm{mV} / \mathrm{cm}$ and \pm 100 $\mathrm{mV} / \mathrm{cm}$. When the step voltage was at $\pm 1000 \mathrm{mV} / \mathrm{cm}$, death of RGCs was visible since the nucleus became very small and the cytoskeleton was fragments. This could be attributed to the over-polarization of cells under strong electrical field. When the 
step constant potential was pulsed between $-700 \mathrm{mV} / \mathrm{cm}$ and $+700 \mathrm{mV} / \mathrm{cm}$, growth of RGCs increased significantly. $\pm 700 \mathrm{mV} / \mathrm{cm}$ was thus chosen as the step potential for electrical stimulation.
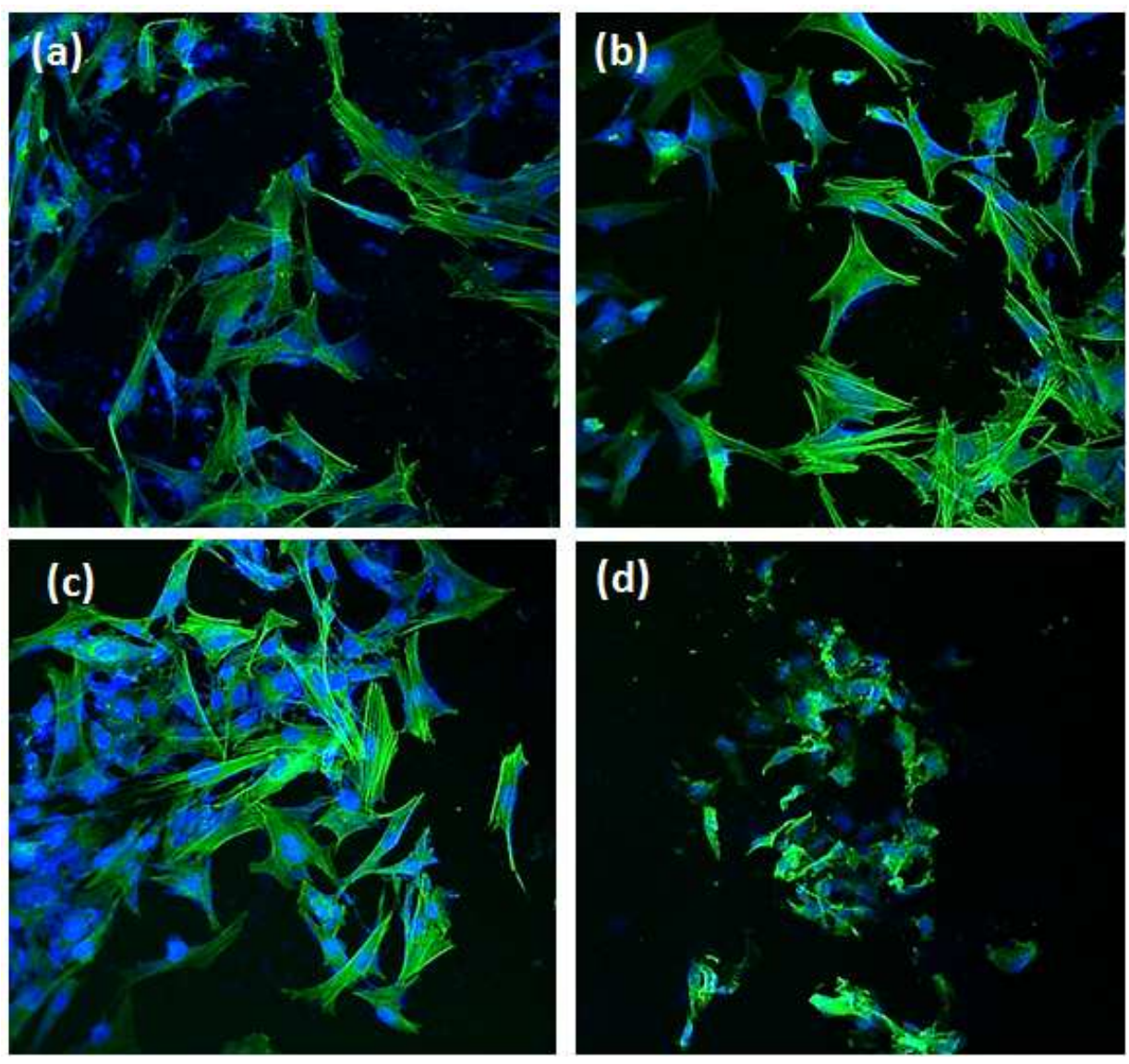

Figure S5. Confocal micrographs of RGCs on the ITO glass after electrical stimulation when the step constant potential was pulsed between (a) $-100 \mathrm{mV} / \mathrm{cm}$ and $100 \mathrm{mV} / \mathrm{cm}$, (b) $-400 \mathrm{mV} / \mathrm{cm}$ and $400 \mathrm{mV} / \mathrm{cm}$, (c) $-700 \mathrm{mV} / \mathrm{cm}$ and $700 \mathrm{mV} / \mathrm{cm}$, (d) $-1000 \mathrm{mV} / \mathrm{cm}$ and $1000 \mathrm{mV} / \mathrm{cm}$. 


\section{Electrical Stimulation on the 6\% PPy aligned nanofibers}

For the purpose of comparison, 6\% PPy was mixed together with PLGA and electrospun into aligned nanofibers at the same conditions. RGCs were seeded onto the PPy/PLGA aligned nanofibers for electrical stimulation. After ES under the same conditions, changes on the cell morphology were shown in Figure S6. As can be seen, cell length hasn't changed too much. Detailed cell length data were shown in Figure $3(d)$.
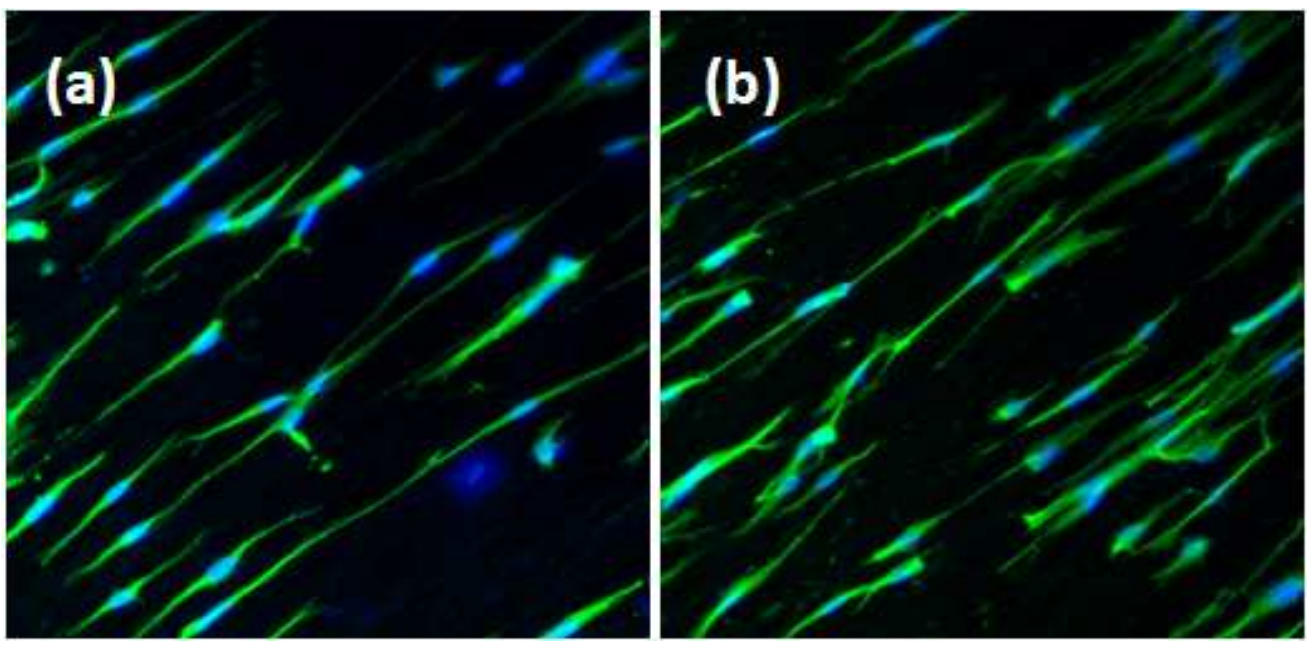

Figure S6. Confocal microscopy images of RGC cells seeded on (a) the aligned $6 \%$ (w/w) PPy/PLGA without ES and (b) after ES.

\section{Scanning electron microscopy}

For SEM analyses, samples were fixed with $2.5 \%$ gluteraldehyde for $2 \mathrm{~h}$, and followed by dehydration with increasing concentrations of ethanol $(30 \%, 50 \%, 70 \%$, $80 \%, 90 \%, 100 \%$ ) for 15 min each, respectively. Finally, samples were dried in the room temperature overnight and ready for SEM imaging.

SEM images of RGCs seeded on the random and aligned PPy-G/PLGA nanofibers 
and RGCs were shown in Figure S7. Promoted cell growth and density after ES was observed at the both types of nanofibers while longer cell length was found at the aligned nanofibers, suggesting that ES could enhance regeneration of RGCs and better ES effects were obtained with the use of aligned fibrous substrate.
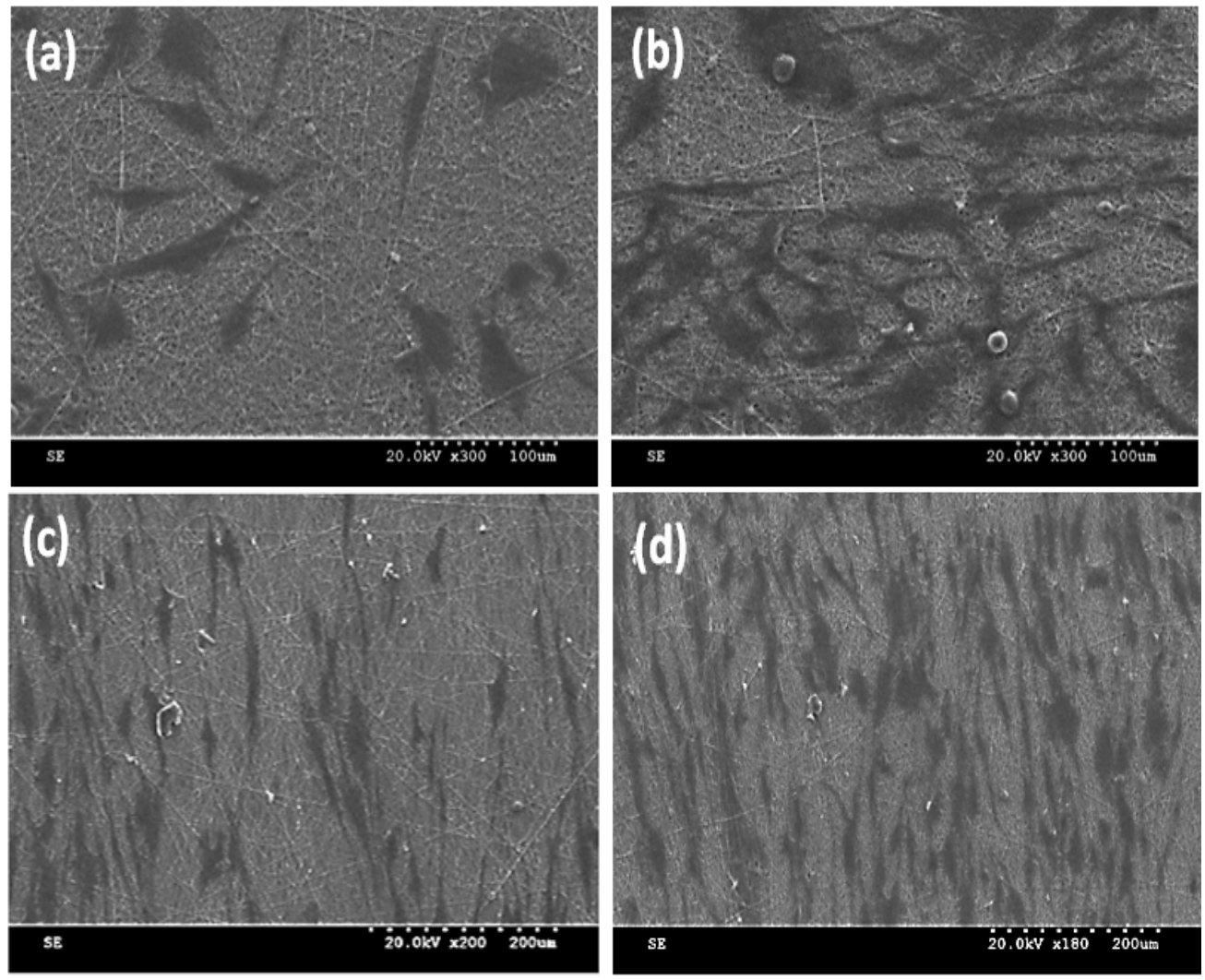

Figure S7. SEM micrographs of RGCs (a) on the random 6\% PPy-G/PLGA nanofibers without ES compared to (b) that after ES; (c) on the aligned $6 \%$ PPy-G/PLGA nanofibers without ES compared to (d) that after ES. 


\section{Immunofluorescence stain of RGCs}

SD rat primary RGCs were used in all in vitro studies. Before cell culturing, the electrospun PPy-G/PLGA nanofibers were dried using vacuum dryer for 2 days and sterilized by UV radiation for $2 \mathrm{~h}$. The nanofibers on the ITO substrates were plated in the 24 well-plate and washed with PBS for 2 times, followed by incubation in DMEM medium for 2 h. 20,000 cells were seeded on the PPy-G/PLGA nanofibers and cultured in DMEM medium which supplemented with 10\% FBS and 1\% penicillin streptomycin. Cells were allowed to grow for $24 \mathrm{~h}$ prior to immunofluorescent staining.

In order to confirm that the isolated cells from SD rats were pure RGCs, the specific antibody of Thy-1 and Brn-3 were used for immuofluorescent staining. Cells in the plate were washed by PBS for 2 times and fixed with pre-cold $4 \%$ paraformaldehyde for 15 min. For Brn-3 antibody staining, 0.1\% Triton-x 100 was added for cell membrane permeabilization for $5 \mathrm{~min}$. 3\% BSA solution was used to block un-specified proteins at room temperature for $1 \mathrm{~h}$. Primary antibody (Thy- 1 and Brn-3) was then introduced to incubate with the cells for $2 \mathrm{~h}$, followed by washing with PBS for 10 min and repeated 3 times. Second antibody was incubated with cells for $1 \mathrm{~h}$ at $37 \square$. PBS was then used to wash it for $10 \mathrm{~min}$ and repeated for 3 times. The immunofluorescence results (Figure S8) confirmed that the as-received cells were pure RGC cells. 

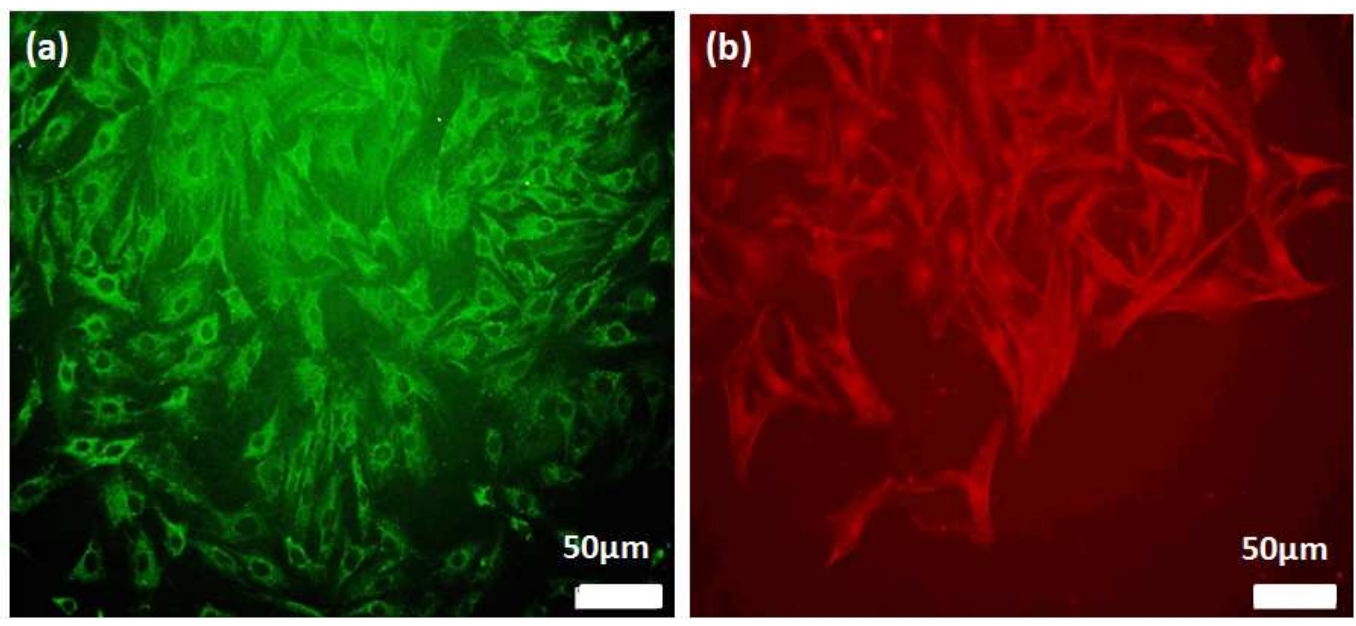

Figure S8. Immunofluorescence stain of RGCs with (a) Thy-1antibody and (b) Brn-3 antibody.

\section{Cytoxicity of the PPy-G with RGC cells}

RGCs were seeded at the density of 5,000 cells/well in the 96-well plate. $24 \mathrm{~h}$ were allowed for cell spreading. Different amounts of as-synthesized PPy-G nanosheets were sterilized at high temperature, and then added to cell wells. After being co-cultured over $24 \mathrm{~h}$, RGCs were washed using PBS. CCK-8 was subsequently added in. The cell viability results were shown in Figure S9. All cell viabilities were found to be higher than $90 \%$ even the concentration of PPy-G was $100 \mu \mathrm{g} / \mathrm{mL}$, indicative of the excellent biocompatibility of PPy-G. 


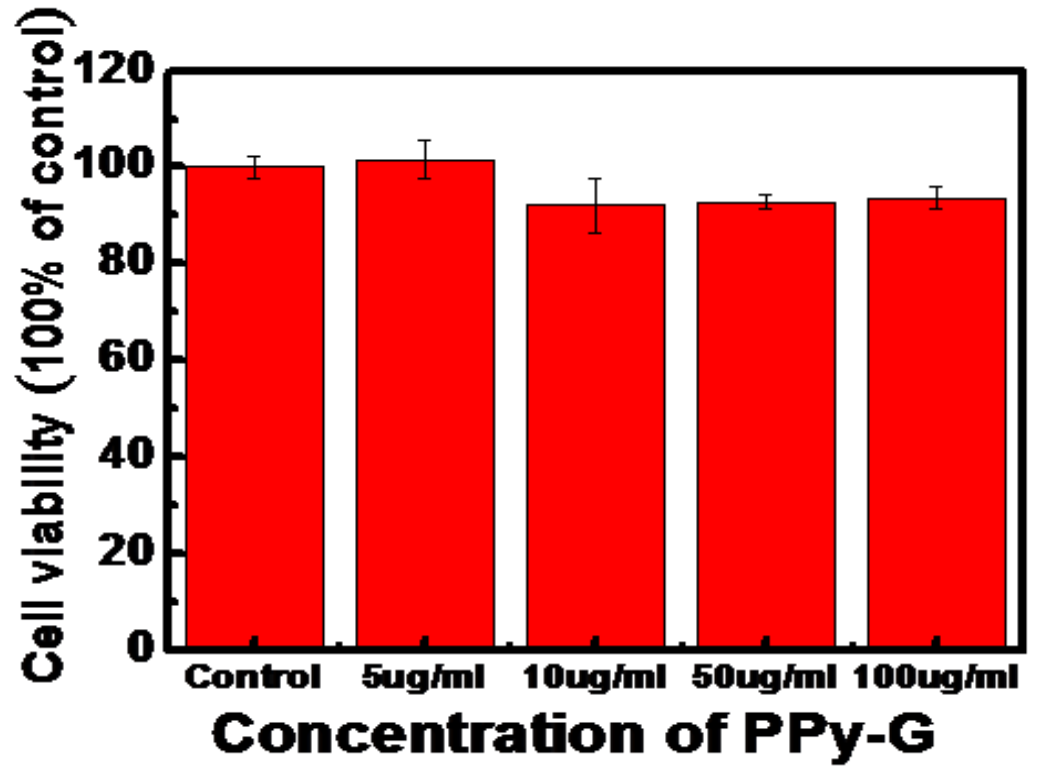

Figure S9. Cell viabilities of RGCs incubated with various amounts of the PPy-G over $24 \mathrm{~h}$

Electrical stimulation set-up

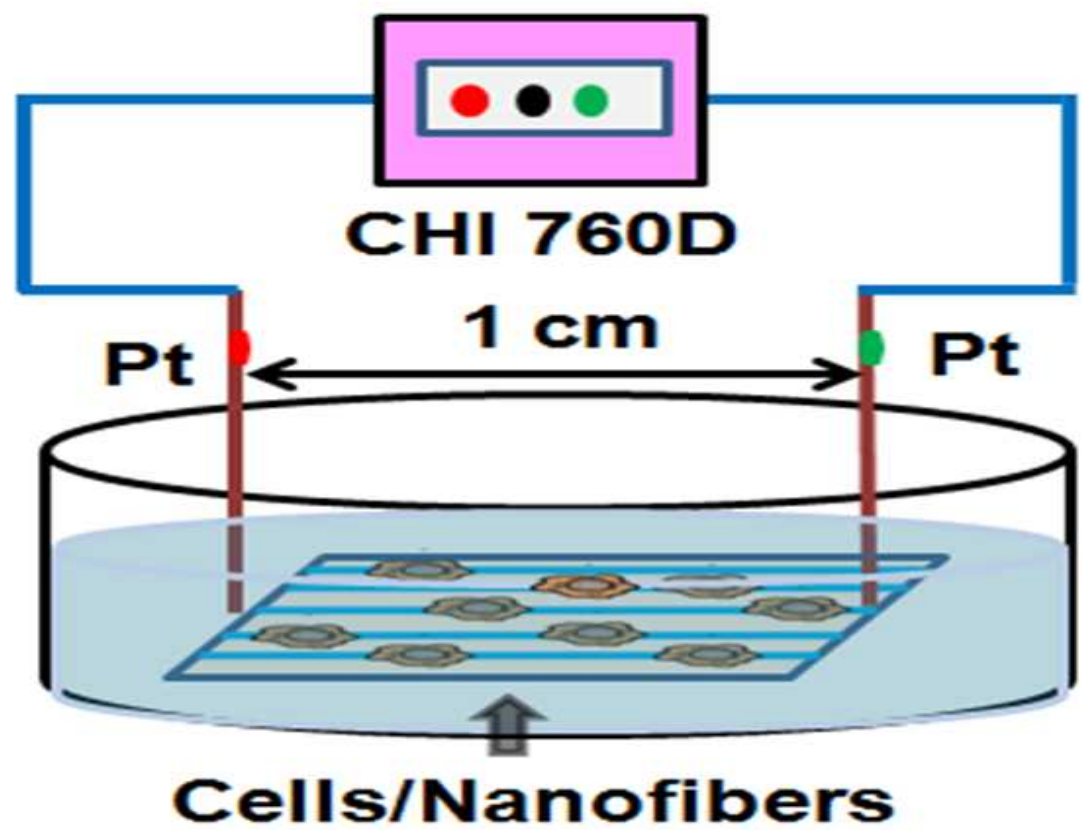

Figure S10. Schematic diagram of the electrical stimulation set-up. 